\title{
Tasks and structure of the WENDELSTEIN 7-X control system
}

\author{
Jörg Schacht ${ }^{*}$, Helmut Niedermeyer, Heike Laqua, Anett Spring, Steffen Pingel, \\ Andreas Wölk
}

Max-Planck-Institut für Plasmaphysik, Teilinstitut Greifswald, Wendelsteinstr. 1, 17491 Greifswald, Germany

\begin{abstract}
The super conducting stellarator WENDELSTEIN 7-X will run pulses of up to 30 minutes duration with full heating power. Short pulses with arbitrary intervals, steady state long discharges and arbitrary sequences of short phases with different characteristics in one discharge will be supported by the control system. Each technical component and each diagnostic system including its data acquisition will have its own control system permitting autonomous operation for commissioning and testing. During the experimental sessions the activity of these devices will be coordinated by a Central Control System and the machine runs more or less automatically with predefined programs. A session leader program allows the leader of the experiment to choose and chain predefined segments, to start or stop a segment chain as a discharge. The progress of the discharge is shown by a sequence monitor attached to the central sequence controller and the session leader program.

W7-X has to be prepared for the experiment and monitored by means of the PLC based operational management system. A safety system working independently of the operational management consists of local units responsible for the safety of each component and a central unit ensuring the safety of the whole W7-X system. This safety system provides interlocks and controls the human access to the device. A safety analysis is the basis for the development of the safety system.
\end{abstract}

\section{Introduction}

The experiment WENDELSTEIN 7-X is a fusion device, based on the stellarator principle, which will start operation in 2011. The magnetic field for the plasma confinement will be produced by 50 non planar and 20 planar superconducting coils. This allows in contrast to most of the other fusion devices pulses with a steady state operation of up to 30 minutes with full heating power or even more with lower power. The goal for this experiment is to explore the reactor potential of a stellarator fusion device [1]. The large discharge time permits different designs of the experiments. The experiments can be planned as short pulses with arbitrary intervals, steady state long discharges and arbitrary sequences of short phases with

* Corresponding author. Tel.: +49-3834-88-2761;

fax: +49-3834-88-2509.

E-mail address: joerg.schacht@ipp.mpg.de (J. Schacht). 
different characteristics in one discharge. It is possible to divide a long discharge into series of short experimental sections, so called segments. During each segment a segment description defines completely the behavior of the components involved in the experiment. Segment descriptions are prepared as database objects by means of a special editor. From this database information software objects are created in local segment control units in advance. In real time the components are controlled by means of these objects. Thus the planned plasma discharge consists of a chain of predefined segments to be carried out in real time. The so called segment processing guarantees a flexible planning and processing of segment programs [2].

The WENDELSTEIN 7-X control system will handle all technical components of the device involved in the different activities for commissioning, tests, preparation and processing of experiments. In order to use the discharge time efficiently and to avoid operator and systems mistakes, the control system will be as automated as possible. Standard procedures, the processing of the experiments during a discharge, the error detection, and if possible the recovery from error states will be performed without need of an active operator intervention.

The technical base of the experiment WENDELSTEIN 7-X is very complex and can be controlled by numerous parameters. Settings of the machine produce the plasma as required, settings of the diagnostic systems and data acquisition ensure, that all necessary measurements are done properly.

The machine will operate for two decades and the experiment has to grow in this time. The commissioning of the device is never completed, new components will be added, and others will be modified or extended. A hierarchical structure of the components and a modular design of the control system facilitate modifications of this kind.

Another important requirement to the experiment control system is an easy and simple use of the technical components by the users.

WENDELSTEIN 7-X has a high potential of hazards. First of all any danger is minimized by a safe design including properly designed passive protection systems. Remaining hazards are handled by interlocks and control of the human access to the device provided by the safety control system.

\section{Architecture and tasks of the control system}

\subsection{System overview}

From the hardware point of view the W7-X device control system consists of a central control system, a large number of local control systems, a data base system and a Trigger Time Event system (TTE system). The local control systems at the bottom of Fig.1 are individually designed for each technical component like coils, heating system, cooling system etc. or scientific component like diagnostics.

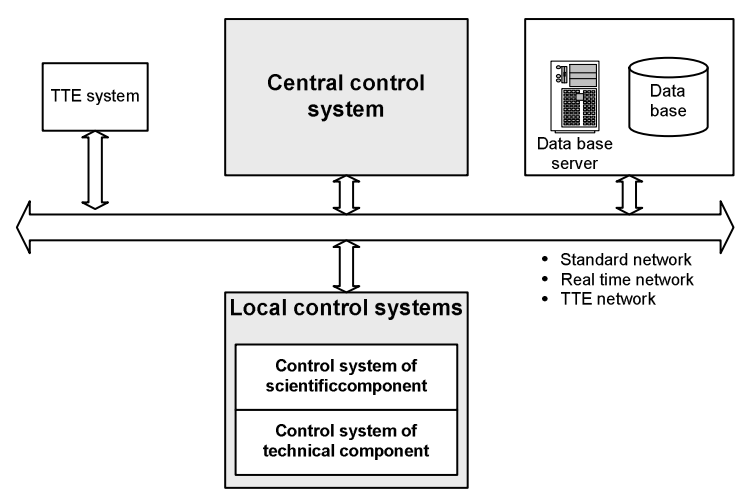

Fig. 1. Generic structure of the W7-X device control system.

The local control components have a few features in common necessary for coordinated operation. The number of local control components is flexible, not limited, and depends of the actual stage of expansion of the experimental device and infrastructure. One central control system coordinates the control systems of the components during experiments or other phases requiring operation of the whole device. In this mode of operation local components are sub-ordinate to the central control system. The device is run from a central control room. Some of the technical components in addition have their own local control rooms, e.g. the magnet system and the heating systems. The TTE system is used for synchronization of all control and data acquisition components of the experiment and for processing of event messages.

All local control components are able to run in a stand-alone mode. This mode is mainly used for commissioning, tests and preparation of the 
components for automatic control.

For segment processing the local components have to be set into the subordinate state. A standard software module implements the mechanism for switching between stand alone (the component is not subordinate) and slave mode (the component is subordinate). The segment descriptions in this case define the behavior of the control components. Segment processing is the standard mode of the components during an experimental phase; however it can also be used in the stand alone mode for testing.

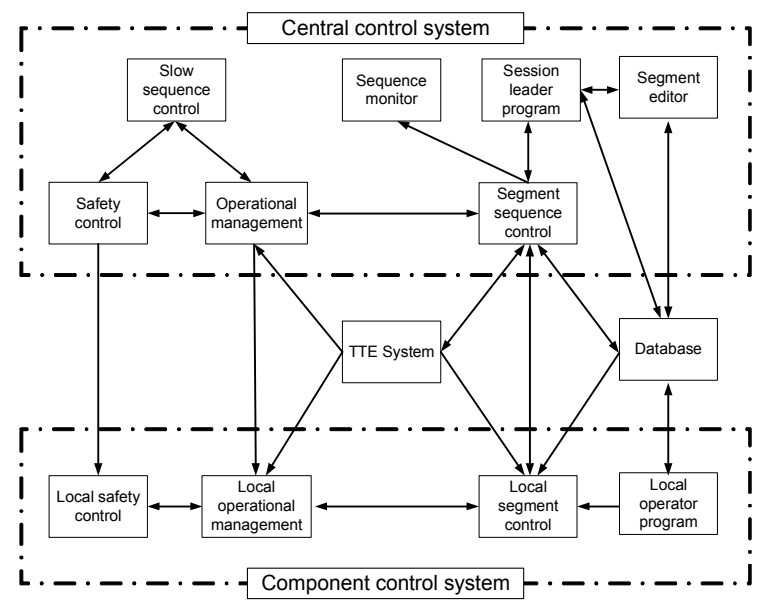

Fig. 2. Subcomponents of the device control system.

The substructure of the device control system is shown in Fig.2. The control system of the W7-X has a hierarchical, tree-like structure. On the top of this structure is located the central control system. Nodes of the structure are built by the component control systems and their subsystems. The chosen hierarchy allows a high independence between the local components and makes an easy control possible during the segment processing.

If necessary each component has its own safety system. This system guarantees the safety of personnel and environment for the isolated system. The safety system of the central control system handles interactions between components, the environment, and personnel. It provides enable signals for the components depending on the machine state like standby, experiment etc. and interlocks between components.

The segment editor, session leader program, segment sequence control and the sequence monitor are essential for defining, processing of segment processing and execution monitoring. Local components are equipped with one or more local segment control units responsible for generating the control objects, switching between these objects in real time and controlling the component by means of the active objects.

The component operational management is responsible for the preparation of the components and the whole system.

Some technical processes, e.g. baking and conditioning of the vessel, are realized by the slow sequence control. The processes of the slow sequence control are characterized by long time constants and relatively static sequences with predefined parameter sets. The components slow sequence control is strongly related to the operational management.

Standard products are used for the realization of different kinds of control. For slow control processes the Siemens SIMATIC S7 PLCs (programmable logic controller) is used. For visualization of these systems the Siemens WinCC program in a client-server configuration has been chosen. Control processes with real time requirements are done on standard PCs in 19 ' casing running the VxWorks operating systems. Real time software is being developed in $\mathrm{C}++$. Programs for interaction with the user like the session leader program and the segment editor are written in JAVA to be independent of the operating system.

The TTE system is a proprietary development because no adequate commercial solution has been found on the market.

\subsection{Tasks of the control components}

In the following sections the tasks of the parts of the control system shown in Fig. 2 are outlined.

\subsubsection{Control of operational states and safety tasks}

For the experiment W7-X a set of six basic system states has been defined:

1. Emergency stop,

2. Switched off,

3. Standby,

4. Experiment pause,

5. Experiment operation, and 
6. Partially protected operation.

Each system state defines hazard potentials of the whole device. These system states provide a basis for design and definition of safety systems reactions. For example, the access to the torus hall and to the plasma vessel will be defined on the base of the actual system state. The state Emergency stop is a special state. The transition into this state can be enforced from all others states at any time by pressing one of many emergency stop buttons. At a transition to this state the emergency stop of all components of the whole system is invoked. The state Emergency stop can only be left after a manual acknowledgment first in the central safety system and then in all component control systems. In the state Switched off all components with a hazard potential are switched off and locked. This state will be chosen for long assembly or rebuilding phases. The torus can be open, free access to the torus hall and controlled access into the plasma vessel can be allowed.

The Stand by state is used for pauses of several hours up to a few days. The torus is closed and can be vented or evacuated. The vacuum system and the cooling system of the cryostat are active. Free access to the torus hall can be allowed.

During preparation of an experiment and in short experimental pauses the state Experiment pause is active. Components with a complex hazard potential like high power lasers or ECRH cannot be activated. The magnetic field can be energized at a static level. The torus vessel is closed and evacuated. Controlled access to the torus hall may be allowed for minor activities under certain restrictions.

The Experiment operation is the standard state for running physical and technical experiments. All necessary components of the control and data acquisition systems are normally switched on and can be activated. Experiments can be controlled by the segment control system in a more or less automatic manner. A session leader selects the predefined segment programs for the experimental phase and starts and supervises the processing of these segments. The torus hall is not accessible.

The last operational state is the Partially secured operation. This state is necessary for a few activities when access to the torus hall is necessary despite of existing hazards, for example for aligning laser beams. Individual interlocks can be by-passed in this state.
Access may be allowed to trained staff well aware of the hazards and wearing personal safety equipment. The components are responsible for handling safety related problems as far as this is possible on the component level. The central control system ensures safety of interactions between the components of the whole experiment and safety of persons in the experiment hall. A set of standard safety signals has been defined for the communications between the safety systems of the components and the central safety system. The central system sends emergency stop and enable signals to the local control components. The components confirm that they are in the state emergency stop or in a safety state, respectively. If the central safety system does not enable a component, then any activities with a hazard potential inside the torus hall are prevented. Deactivation of the enable signal by the central safety system during operation enforces a transition of the component into a safe state. The local components confirm their safe state and prevent any hazardous activities in order to protect personnel, other components and the environment.

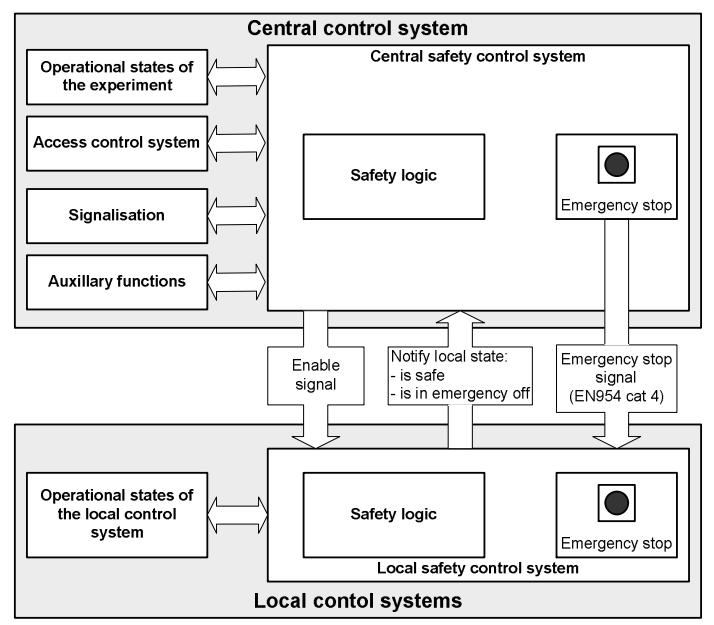

Fig. 3. Structure of the safety system

The structure of the safety system of the whole experiment is shown in Fig. 3. Other components of the central safety system are the signalization and a set of auxiliary functions. For the public signalization of safety related states like the system states described above a set of hardware signal units and large hardware displays will be used. A safety monitor based on Siemens WinCC provides detailed information about 
the safety system to the operator.

The gas monitoring system, the hall exhaust system and the fire alarm system are autonomous systems with defined interfaces to the safety system.

The central safety system will be implemented by a safety PLC SIMATIC S7-400 F/H. Optical links running fail-safe Profibus and Ethernet protocols and hard wired connections in cat 4 safety technique will be used for interfaces between the components and the central control system.

\subsubsection{Tasks of the operational management}

For running an experiment in addition to the local components (e.g. magnet system, vacuum system, plasma heating system, cryogenic system, ...) infrastructure (e.g. power supplies for the control equipment, uninterruptible power supplies, light control in the torus hall, communication systems) is necessary. The main task of the central operational management is the supervision and the visualization of all components of the experiment as well as the infrastructure. The central operational management also allows limited manual operation of components in slave mode, for example, switching-on a simple diagnostic which does not need a special operator.

Important operational parameters of the whole device are collected and visualized. The central operator is informed on time about the currents of the superconducting magnet coils, the power of the plasma heating systems etc. The visualizations show first a summary of the status of all components. This includes the operational state of the components, the control mode (stand alone or slave), and status of alarms and warnings. If more detailed information is needed the operator can choose more specific visualization windows.

The torus hall will be observed during an experiment. The noise from the torus hall is recorded and video cameras record the situation in the torus hall. Acoustic messages can be sent out automatically under predefined conditions or on request.

\subsubsection{Task of the slow sequence control}

Processes for the preparation of the experiments like glow discharge cleaning have long physical and technical time constants. Their control processes are running in well known and predefined sequences using sets of control parameters or manual control. The slow sequence control system which is independent of the segment control system coordinates the activities of all components necessary for these processes. Not offering the flexibility of the segment control system the slow sequence control is much simpler to use. Visualization informs the operator about the actual status of the control sequence.

\subsubsection{Segment processing tasks}

During experimental operation the session leader starts and monitors the processing of experiment programs, so called discharge programs. The live cycle for discharge programs is shown in Fig. 4. The first step is the preparation of segment descriptions by experts. For segment planning the experts use a special editor [3] and store the generated segments in a database. The editor allows both producing new segment descriptions in a comfortable way and copying suitable proven segment descriptions and modifying small parts of them as required. For preparing reasonable physical discharge programs a sequence of segments has to be enforced. By using the editor program the user can chain up the segments to build segment chains. In a separate process the sequences of segments will be checked by a software program module "segment checker". The check is based on the certain physical, technical and logical rules for the individual segments and for the chaining the segments.

The planning of a session requires the arrangement of a choice of predefined segment chains into a discharge program. The session leader program [4] is used for this task. This program has functions for checking the feasibility of the proposed segment programs in the actual state of the device. The chosen segments are marked as so called active segments. Continuously and on request all segment control computers read the list of these active segments and generate the necessary data objects from the segment descriptions stored in the database [5].

A validated discharge program will be sent to the segment sequence controller as an ordered list of segment identifiers.

On the level of the session controlling the session leader starts the automatic processing of the planned segment programs. 


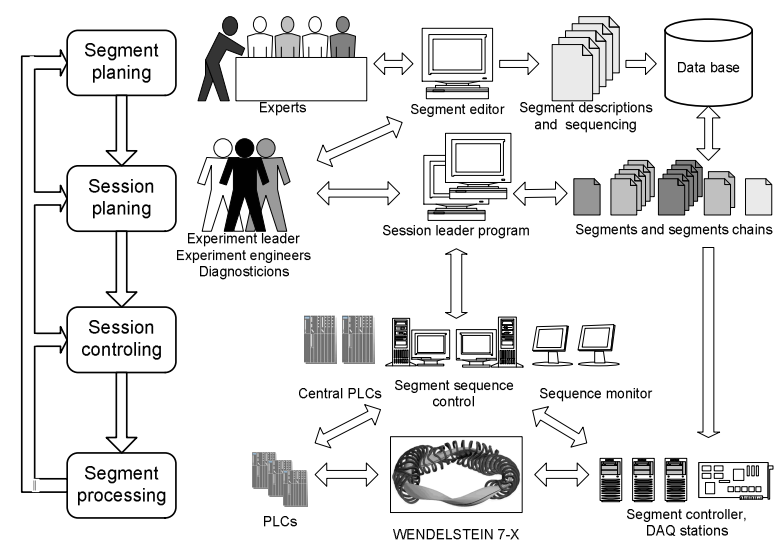

Fig. 4 Segment processing.

The central segment sequence controller triggers all local control computers to synchronously process the segments within the discharge program. During segment processing the session leader can interactively manage the sequence controller at any time, e.g. skip segments or stops the discharge.

If a local control system works in stand alone mode the same program can be used as a local operator program for interactive control of segment processing. In this case all commands from the central sequence control system are ignored.

\subsubsection{Tasks of the Trigger Time Event system}

The control system demands synchronizing processes in many distributed technical components with accuracy from milliseconds to nanoseconds. For this purpose the TTE system was developed [6]. Its main task is the synchronization of all control components and data acquisition systems. It allows time stamping, processing of trigger signals and event messages. The TTE system offers the possibility to program timing sequences and delays, logic combinations or conditions.

The real time segment controllers are equipped with a standard timing system based on a local TTE computer card. This timing system provides periodic cycles for synchronous and asynchronous segment processing [7]. Segment control needs a precise common start time for different distributed tasks, for example to switch to a segment in all segment control components at the same time. All data from diagnostics and control systems are acquired synchronously throughout $\mathrm{W} 7-\mathrm{X}$ in order to ease direct comparison and are precisely time stamped.

\section{References}

[1] G. Grieger and the W7-X Team, The Wendelstein 7-X project, J. Plasma Fusion Research 1 (1998) 53-56.

[2] H. Laqua, H. Niedermeyer, and J. Schacht, Control system of WENDELSTEIN 7-X experiment, Fusion Engineering and Design 66-68 (2003) 669-673.

[3] H. Kühntopf and the XDV Team, Specialized editor for processing objects in a database to prepare discharges for WENDELSTEIN 7-X, Fusion Engineering and Design this issue

[4] A. Spring, H. Laqua, H. Niedermeyer, User interaction concept for plasma discharge control on WENDELSTEIN 7-X, Fusion Engineering and Design this issue

[5] H. Laqua, H. Niedermeyer, J. Schacht, and A. Spring, Real Time Software for the Fusion Experiment WENDELSTEIN 7-X, Fusion Engineering and Design this issue J. Schacht, H. Niedermeyer, Ch. Wiencke, J. Hildebrandt, and A. Wassatsch, A trigger-time-event system for the W7-X experiment, Fusion Engineering and Design 60 (2002) 373-379.

[6] J. Schacht, H. Laqua, and H. Niedermeyer, Synchronization of processes in a distributed real time system exemplified by the control system of the fusion experiment WENDELSTEIN 7-X, Proc. 14th IEEENPSS Real Time Conference, Stockholm, Sweden, 2005. 